\title{
Seismic trace interpolation in the $f-x-y$ domain
}

\author{
Yanghua Wang*
}

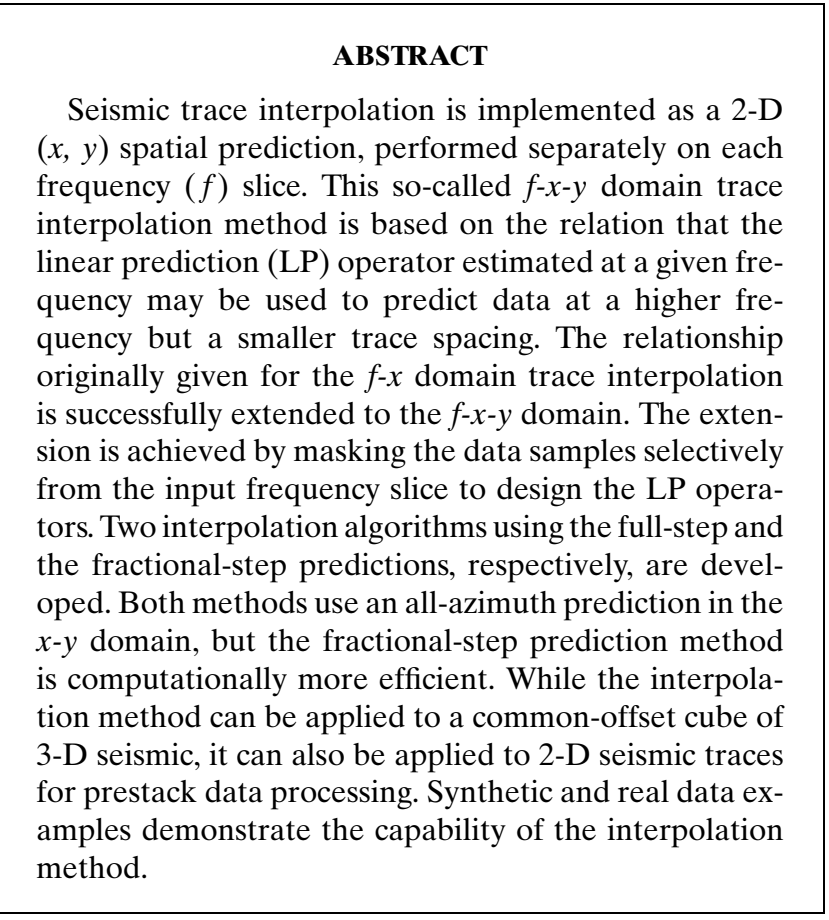

\section{INTRODUCTION}

The resolution of seismic imaging depends in high degree on the spatial sample interval. Too large a spatial sample interval leads to an aliasing problem which adversely affects migration and results in poor lateral resolution of the subsurface image. It is often the case, however, that seismic data sets are spatially undersampled. In 3-D seismic, for example, the spatial interval between binning lines is typically two or three times greater than the trace interval along the line. To ultimately improve the image resolution, it is necessary to generate unrecorded seismic traces from a spatially undersampled data set (e.g., Larner et al., 1981; Bardan, 1987; Spitz, 1991; Claerbout, 1992; Canales and Lu, 1993; Porsani, 1999).
One of the interpolation methods is called the $f-x$ domain trace interpolation method (Spitz, 1991; Porsani, 1999). For a seismic data set presented in the frequency $(f)$ domain, trace interpolation employs linear prediction (LP) theory acting on each frequency independently. Spitz (1991) used the full-step LP theory; Porsani (1999) used the half-step LP theory based on the predictability of linear events in the spatial $(x)$ domain. This $f-x$ domain linear prediction method is a model-free trace interpolation method that requires no knowledge of dips of events that may be linear in the time-space domain. The method has been used extensively in routine data processing to interpolate spatially aliased 2-D seismic data before stacking or migration, regardless of the spatial interval of the input data set as long as it is regularly sampled in space.

The $f-x$ domain interpolation method is extended in this paper to the $f-x-y$ domain. When the method is applied to $3-\mathrm{D}$ seismic, the two spatial axes are defined by the in-line and cross-line directions in a common-offset (or zero-offset stack) cube. The method may also be applied to 2-D seismic traces for prestack data processing, where one spatial axis represents the source-receiver offset and the other is, for example, the gather number of shots, receivers, or midpoints. The interpolation problem can then be specified as follows: given a 3-D data cube regularly sampled in the spatial directions with intervals $\Delta x$ and $\Delta y$, interpolate traces in the $x$ direction with the interpolation factor $\lambda$, typically 2 or 3 . After interpolation (as shown in Figure 1), the trace intervals are $\Delta x / \lambda$ and $\Delta y$.

Trace interpolation is accomplished in two stages: estimating the LP operator from the original data set, and applying the operator with modification to interpolate seismic traces. If the two LP operators in the estimation and interpolation stages are shown as vectors $\mathbf{p}$ and $\tilde{\mathbf{p}}$, respectively, trace interpolation exploits the following relation:

$$
\tilde{\mathbf{p}}(f)=\mathbf{p}(f / \lambda)
$$

That is, the prediction operator estimated at a given frequency may be applied to predict data at higher frequency but smaller trace spacing. The relationship, given by Spitz (1991) originally for spatial interpolation in one dimension, is extended

Manuscript received by the Editor May 30, 2000; revised manuscript received December 26, 2001.

*Robertson Research International Ltd., Horizon House, Azalea Drive, Swanley, Kent BR8 8JR, United Kingdom. E-mail: yanghua@geo. robresint.co.uk.

(C) 2002 Society of Exploration Geophysicists. All rights reserved. 
in this paper for performing an all-azimuth linear interpolation in the $x-y$ plane. To achieve this extension, I propose to mask seismic traces selectively in the LP operator estimation stage. Two interpolation methods that use the full-step and the fractional-step prediction theorems, respectively, are described in the following sections.

\section{THE FULL-STEP PREDICTION METHOD}

\section{Summary}

The full-step LP system is depicted in Figure 2, where each trace $u_{m, n}$ is predictable (in the least-squares sense) from surrounding traces by using a $L \times L$ operator $(L=5$, for exam-

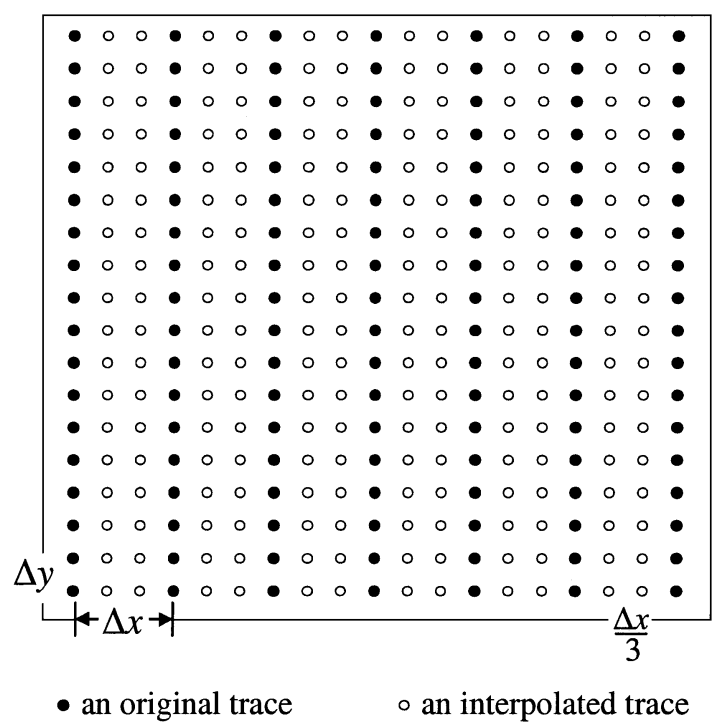

FIG. 1. Example of trace interpolation in the $x$ direction. The original data set has trace interval $\Delta x \times \Delta y$. After interpolation, the trace interval becomes $\Delta x / \lambda \times \Delta y$, where $\lambda$ is the interpolation factor $(\lambda=3$ in this example).

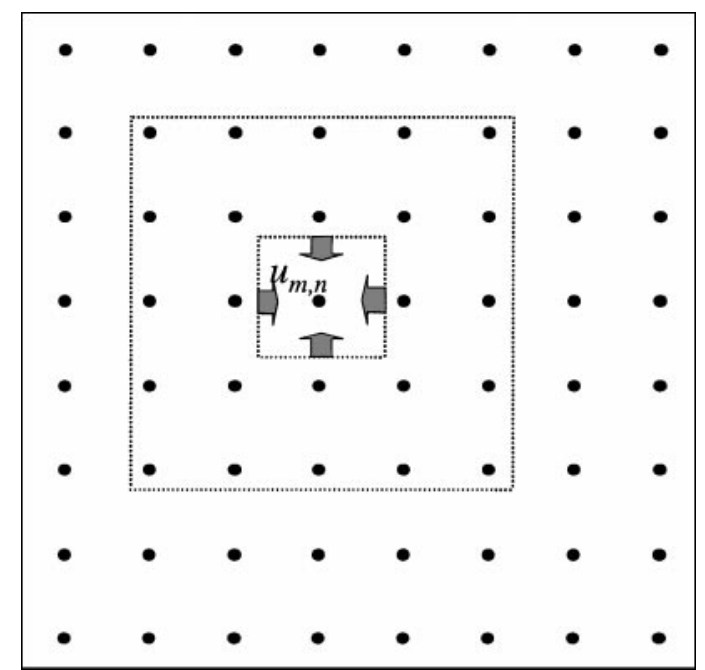

FIG. 2. Full-step linear prediction. Each trace $u_{m, n}$ may be predicted from surrounding traces by using a $L \times L$ prediction operator $(L=5$ in this example). ple). As the step size between the traces to be predicted $\left(u_{m, n}\right)$ and the adjacent traces $\left(u_{m-1, n}\right.$ or $\left.u_{m+1, n}\right)$ is equivalent to the sample interval in the prediction direction, this interpolation is referred to as full-step prediction method.

Representing a plane event with apparent time shifts $\Delta \tau_{x}$ and $\Delta \tau_{y}$ between adjacent traces in the $x$ and $y$ directions by

$$
w(f)=a(f) e^{i 2 \pi f \sqrt{\left(m \Delta \tau_{x}\right)^{2}+\left(n \Delta \tau_{y}\right)^{2}},}
$$

where $a(f)$ is the amplitude and the exponential term describes the relative phase of the $(m, n)$ th trace with respect to the $(0,0)$ th trace, the datum $u_{m, n}(f)$ is modeled by the sum of a number of such dipping events. In linear prediction theory, one assumes that $a(f)$ is locally invariant in space so that a plane event may be characterized by its phase shift. Then, the analytical expression of the LP operator involves only products of the phase shifts at the corresponding frequency (Spitz, 1991).

To exploit relationship (1) in the full-step LP system, I mask traces selectively in the $y$ direction so as to mimic the trace intervals being $\Delta x$ and $\lambda \Delta y$. For the masked data set where the time shift in the $y$ direction is $\lambda \Delta \tau_{y}$, the phase shift of the $\left(m^{\prime}, n^{\prime}\right)$ th trace against the $(m, n)$ th trace would be

$$
\phi(f)=e^{i 2 \pi f \sqrt{\left[\left(m^{\prime}-m\right) \Delta \tau_{x}\right]^{2}+\left[\left(n^{\prime}-n\right) \lambda \Delta \tau_{y}\right]^{2}}} .
$$

The phase shift within the interpolated cube is, therefore,

$$
\begin{aligned}
\tilde{\phi}(f) & =e^{i 2 \pi f \sqrt{\left[\left(m^{\prime}-m\right) \Delta \tau_{x} / \lambda\right]^{2}+\left[\left(n^{\prime}-n\right) \Delta \tau_{y}\right]^{2}}} \\
& =e^{i 2 \pi(f / \lambda) \sqrt{\left[\left(m^{\prime}-m\right) \Delta \tau_{x}\right]^{2}+\left[\left(n^{\prime}-n\right) \lambda \Delta \tau_{y}\right]^{2}}} \\
& =\phi(f / \lambda) .
\end{aligned}
$$

Thus, relation (1) is satisfied.

Figure 3 illustrates the $f-x-y$ trace interpolation with $\lambda=3$, for example, using this full-step LP theory.

\section{Estimation of the LP operator}

Each LP operator is estimated for a single frequency slice, so explicit reference to frequency is dropped from the following expressions. Given an $M \times N$ frequency slice, the $L \times L$ LP operator may be expressed in terms of filter coefficients $p_{i, j}$ as

$$
\begin{aligned}
& u_{m, n}=\sum_{j=1}^{L / 2} p_{0, j} u_{m, n-\lambda j}+\sum_{i=1}^{L / 2} \sum_{j=-L / 2}^{L / 2} p_{i, j} u_{m-i, n-\lambda j}, \\
& \text { for } m=1+L / 2, \ldots, M \text {, } \\
& n=1+\lambda L / 2, \ldots, N-\lambda L / 2 \text {, }
\end{aligned}
$$

and

$$
\begin{array}{r}
u_{m, n}^{*}=\sum_{j=1}^{L / 2} p_{0, j} u_{m, n+\lambda j}^{*}+\sum_{i=1}^{L / 2} \sum_{j=-L / 2}^{L / 2} p_{i, j} u_{m+i, n+\lambda j}^{*} \\
\text { for } \begin{aligned}
m & =1, \ldots, M-L / 2 \\
n & =1+\lambda L / 2, \ldots, N-\lambda L / 2
\end{aligned}
\end{array}
$$

where $u_{m, n}^{*}$ denotes the complex conjugate of $u_{m, n}$. The two parts of this 2-D all-azimuth LP system may be described, respectively, as a forward prediction and a backward prediction. In the first equation (5) each data sample $u_{m, n}$ is a weighted 
sum of the preceding data, whereas in the second equation (6) each data point $u_{m, n}$ is represented as a weighted sum of the future data. Figure $3 c$ indicates the subsets of points that are used by each of these operators. The prediction directions are shown by arrows towards the output position.

The all-azimuth LP operator is an $L \times L$ square filter, which may be split into the forward and backward prediction operators (Wang, 1999). These divided operators (with $L=5$, for example), as shown schematically in Figure 3c, have the forms of

$$
\left[\begin{array}{ccc}
p_{2,2} & p_{1,2} & p_{0,2} \\
p_{2,1} & p_{1,1} & p_{0,1} \\
p_{2,0} & p_{1,0} & {[\mathrm{x}]} \\
p_{2,-1} & p_{1,-1} & \\
p_{2,-2} & p_{1,-2} &
\end{array}\right] \text { and }\left[\begin{array}{ccc} 
& p_{1,-2}^{*} & p_{2,-2}^{*} \\
& p_{1,-1}^{*} & p_{2,-1}^{*} \\
{[\mathrm{x}]} & p_{1,0}^{*} & p_{2,0}^{*} \\
p_{0,1}^{*} & p_{1,1}^{*} & p_{2,1}^{*} \\
p_{0,2}^{*} & p_{1,2}^{*} & p_{2,2}^{*}
\end{array}\right]
$$

respectively, where the output position is under the "[x]" coefficient in the prediction operators. The two operators have conjugate symmetry, and the resultant all-azimuth $L \times L$ filter has zero phase in the wavenumber space. The spectral properties of such a noncausal spatial prediction filter were described by Claerbout (1992) and Gülünay (2000).

The LP system consisting of equations (5) and (6) may be represented in vector-matrix notation as

$$
\mathbf{D p}=\mathbf{d},
$$

where $\mathbf{D}$ is the data matrix, $\mathbf{p}$ is the forward prediction operator, and $\mathbf{d}$ is the desired output vector. The least-squares solution is given by

$$
\mathbf{p}=\left(\mathbf{D}^{H} \mathbf{D}+\sigma^{2} \mathbf{I}\right)^{-1} \mathbf{D}^{H} \mathbf{d},
$$

where $\mathbf{D}^{H}$ is the Hermitian transpose of matrix $\mathbf{D}, \sigma^{2}$ is the noise expectation, and $\mathbf{I}$ is the identity matrix. Solution (9) assumes that the noise is random and has the same power in every trace. The solution is stabilized by the inclusion of the smoothing operator $\sigma^{2} \mathbf{I}$.

\section{Trace interpolation}

The LP theorem is now applied to the interpolation, where

\begin{tabular}{|c|c|c|c|c|c|c|}
\hline$\bullet$ & $\bullet$ & $\bullet$ & $\bullet$ & $\bullet$ & • & $\bullet$ \\
\hline • & $\bullet$ & $\bullet$ & • & $\bullet$ & $\bullet$ & $\bullet$ \\
\hline$\bullet$ & - & $\bullet$ & $\bullet$ & $\bullet$ & $\bullet$ & $\bullet$ \\
\hline$\bullet$ & $\bullet$ & $\bullet$ & $\bullet$ & • & $\bullet$ & - \\
\hline$\bullet$ & $\bullet$ & $\bullet$ & $\bullet$ & $\bullet$ & $\bullet$ & $\bullet$ \\
\hline$\bullet$ & $\bullet$ & $\bullet$ & - & $\bullet$ & $\bullet$ & - \\
\hline$\bullet$ & $\bullet$ & $\bullet$ & • & - & $\bullet$ & $\bullet$ \\
\hline$\bullet$ & $\bullet$ & $\bullet$ & $\bullet$ & • & $\bullet$ & • \\
\hline$\bullet$ & - & $\bullet$ & $\bullet$ & - & $\bullet$ & - \\
\hline$\bullet$ & $\bullet$ & $\bullet$ & $\bullet$ & • & - & - \\
\hline$\bullet$ & $\bullet$ & $\bullet$ & $\bullet$ & - & $\bullet$ & $\bullet$ \\
\hline$\bullet$ & $\bullet$ & $\bullet$ & - & - & - & $\bullet$ \\
\hline - $\Delta x$ & $\bullet$ & $\bullet$ & $\bullet$ & $\bullet$ & $\bullet$ & $\bullet$ \\
\hline
\end{tabular}
a data sample is denoted by $\tilde{u}_{m, n}$ and a coefficient of the LP a)

c)

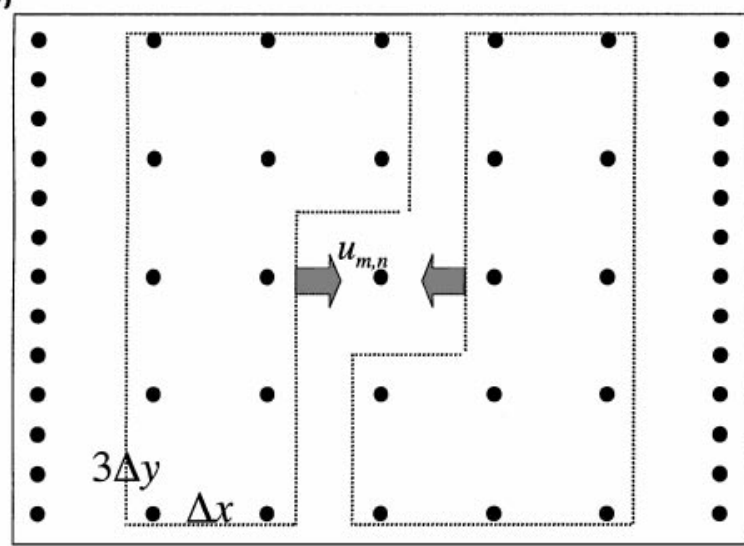

b)

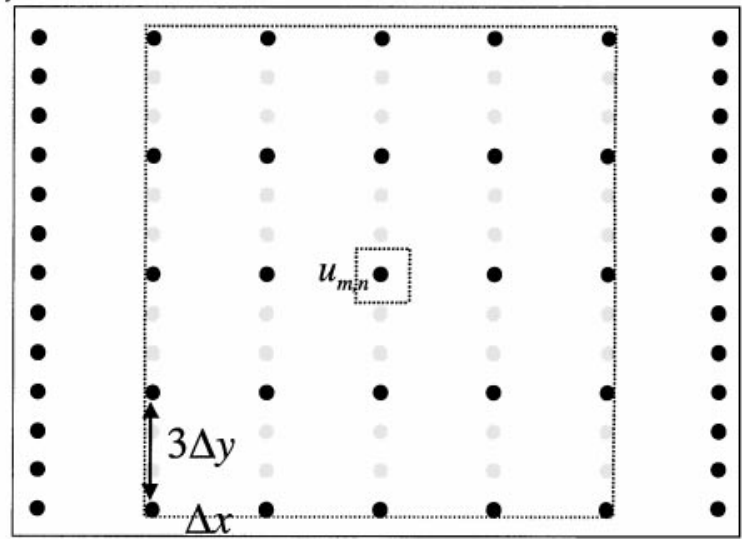

d)

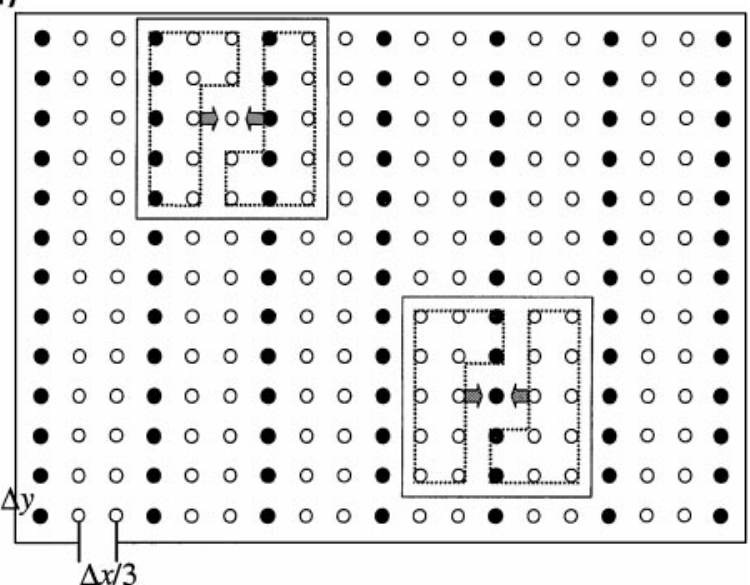

FIG. 3. The full-step prediction method: (a) the original data set with trace interval $\Delta x \times \Delta y$; (b) masking every second and third trace in the $y$ direction; (c) predicting the central trace, using forward and backward predictions; (d) trace interpolation with new trace interval $\Delta x / 3 \times \Delta y$. 
operator by $\tilde{p}_{i, j}$. The operator is obtained from the solution of equation (9), followed by application of equation (1). Given an input $M \times N$ frequency slice, after interpolation its size becomes $\tilde{M} \times N$, where $\tilde{M}=1+\lambda(M-1)$.

In the interpolation stage, the LP filter determined above is applied on a reduced spatial scale (as depicted in Figure 3d) and expressed as

$$
\begin{array}{r}
\tilde{u}_{m, n}=\sum_{j=1}^{L / 2} \tilde{p}_{0, j} \tilde{u}_{m, n-j}+\sum_{i=1}^{L / 2} \sum_{j=-L / 2}^{L / 2} \tilde{p}_{i, j} \tilde{u}_{m-i, n-j} \\
\text { for } \quad \begin{aligned}
m & =1+L / 2, \ldots, \tilde{M} \\
n & =1+L / 2, \ldots, N-L / 2
\end{aligned}
\end{array}
$$

and

$$
\begin{aligned}
& \tilde{u}_{m, n}=\sum_{j=1}^{L / 2} \tilde{p}_{0, j}^{*} \tilde{u}_{m, n+j}+\sum_{i=1}^{L / 2} \sum_{j=-L / 2}^{L / 2} \tilde{p}_{i, j}^{*} \tilde{u}_{m+i, n+j}, \\
& \text { for } m=1, \ldots, \tilde{M}-L / 2 \text {, } \\
& n=1+L / 2, \ldots, N-L / 2 .
\end{aligned}
$$

Among the $\tilde{M} \times N$ data samples $\left\{\tilde{u}_{m, n}\right\}$, there are $(\tilde{M}-M) \times N$ unknown samples. Denoting the unknown samples by a vector $\mathbf{x}$, the linear system may be written

$$
\mathbf{A x}=\mathbf{b}
$$

where $\mathbf{A}$ is a matrix constructed of coefficients $\tilde{p}_{i, j}$, and $\mathbf{b}$ is a vector consisting of products of coefficients $\tilde{p}_{i, j}$ and the known samples.

Equation (12) is an overdetermined system which consists of $(2 \tilde{M}-L+1)(N-L+1)$ linear equations, when the filter size $L$ is defined by an odd number, and of $(\lambda-1)(M-1) \times N$ unknown samples in vector $\mathbf{x}$. It requires significant computational time to solve them simultaneously. To improve the efficiency, I solve equation (12), using a preconditioned conjugate gradient algorithm (Van der Vorst and Dekker, 1988), within sliding windows.

\section{THE FRACTIONAL-STEP PREDICTION METHOD}

\section{Summary}

In this section where I describe the fractional-step prediction method, I consider the case of $\lambda=3$ for the sake of convenience, without loss of generality. The problem may be stated as: given the input data $\left\{u_{m, n}\right\}$, generate interpolated data $\left\{u_{m+1 / 3, n}\right\}$ and $\left\{u_{m+2 / 3, n}\right\}$.

The strategy is presented in Figure 4 schematically. The allazimuth linear prediction is considered as a combination of two linear predictions:

1) To generate $u_{m+1 / 3, n}$, the all-azimuth prediction is implemented as the $1 / 3$-step forward and the $2 / 3$-step backward predictions.

2) To generate $u_{m+2 / 3, n}$, the all-azimuth prediction is implemented, by symmetry, as the $2 / 3$-step forward and the $1 / 3$-step backward predictions.

The fractional step-sizes " $1 / 3$ " and " $2 / 3$ " refer to the distances between an interpolation point $\left(u_{m+1 / 3, n}\right.$ or $\left.u_{m+2 / 3, n}\right)$ and an original sample point $\left(u_{m, n}\right.$ or $\left.u_{m+1, n}\right)$, which are a fraction of the original sample interval $\Delta x$.

To estimate the 1/3-step and the 2/3-step LP operators in the filter design stage, the data samples are masked selectively (Figures 5a and 5b, respectively). Each of these two LP systems built on the masked data sets is an "enlarged" system with spatial size three times as large as the corresponding LP system in the interpolation stage. This means that the phase shift $\phi(f)$ of the masked data set and the phase shift $\tilde{\phi}(f)$ in the interpolation stage satisfy the condition $\tilde{\phi}(f)=\phi(f / 3)$. Therefore, the relation (1) between the LP operator determined from the coarse grid and the interpolation LP operator as applied to the refined grid is satisfied.

\section{Estimation of the LP operator}

Let us now design the $1 / 3$-step and the $2 / 3$-step LP operators separately, although they will be used jointly in the next interpolation stage.

For the 1/3-step LP operator with $L=5$, for example, the masked data set shown in Figure $5 \mathrm{a}$ is in fact a subset of the grid, with sample spacing $3 \Delta x \times 3 \Delta y$. The LP filter may be represented as
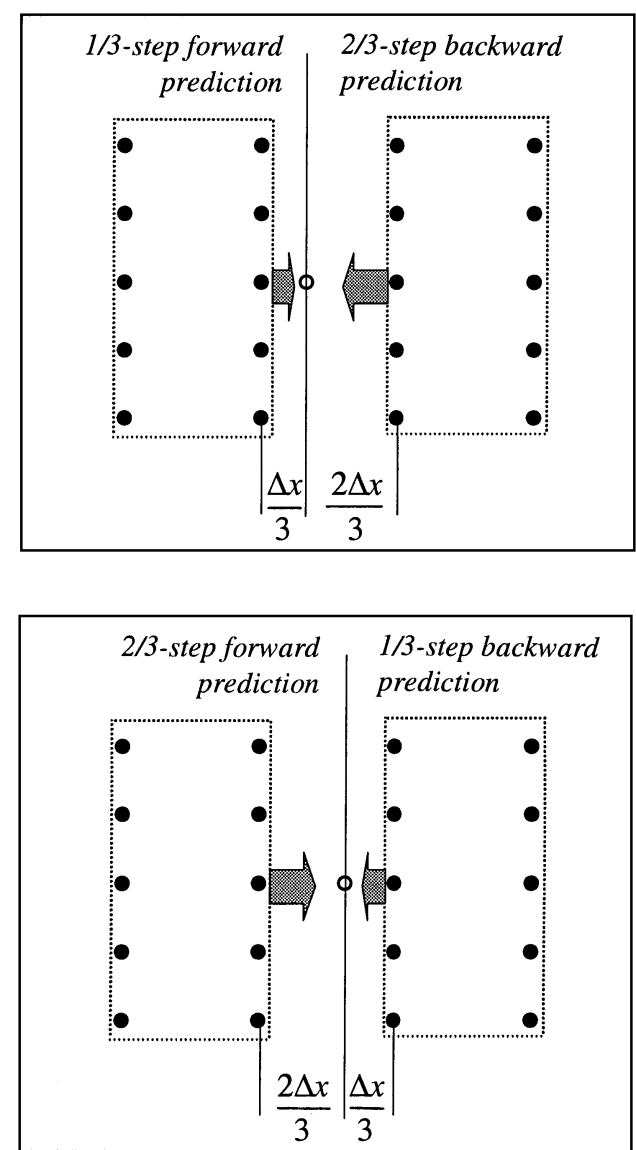

FIG. 4. The fractional step linear prediction method. The all-azimuth prediction is implemented using a forward prediction and a backward prediction, with the 1/3-step and the $2 / 3$-step prediction operators in each case. Filled circle $=$ original trace; open circle $=$ interpolated trace 


$$
\left[\begin{array}{cc}
u_{m-4, n-6} & u_{m-1, n-6} \\
u_{m-4, n-3} & u_{m-1, n-3} \\
u_{m-4, n} & \frac{u_{m-1, n}}{u_{m-4, n+3}} \\
u_{m-1, n+3} \\
u_{m-4, n+6} & u_{m-1, n+6}
\end{array}\right] \rightarrow u_{m, n}
$$

and

$$
u_{m, n} \leftarrow\left[\begin{array}{cc}
u_{m+1, n-6} & u_{m+4, n-6} \\
u_{m+1, n-3} & u_{m+4, n-3} \\
\frac{u_{m+1, n}}{u_{m+1, n+3}} & u_{m+4, n} \\
u_{m+1, n+6} & u_{m+4, n+6}
\end{array}\right]
$$

for the forward and backward linear predictions, respectively. We represent the two data matrices of masked input data, shown in expressions (13) and (14), by vector $\mathbf{a}_{m-1, n}$ and vector $\mathbf{b}_{m+1, n}$, using the indices of the underlined elements $u_{m-1, n}$ and $u_{m+1, n}$ as the reference indices.

The forward and backward LP operators, using the conjugate symmetry, may be represented in the $x-y$ space as

a)

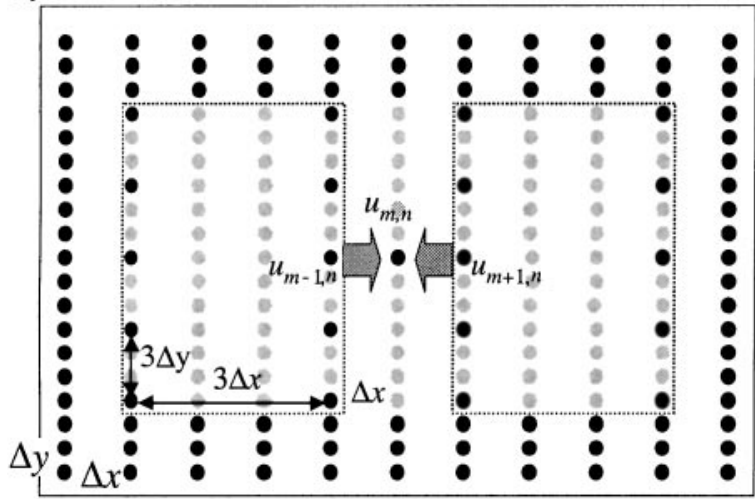

c)

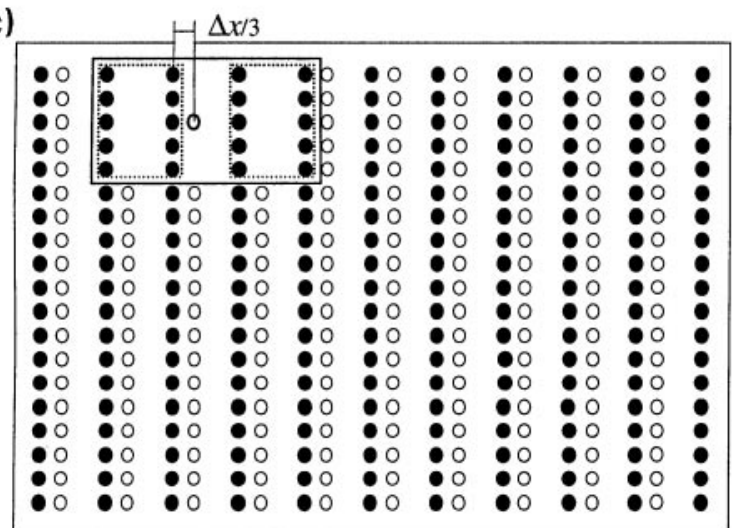

$$
\left[\begin{array}{ccc}
p_{2,2} & p_{1,2} & \\
p_{2,1} & p_{1,1} & \\
p_{2,0} & p_{1,0} & {[\mathrm{x}]} \\
p_{2,-1} & p_{1,-1} & \\
p_{2,-2} & p_{1,-2} &
\end{array}\right] \text { and }\left[\begin{array}{ccc} 
& p_{1,-2}^{*} & p_{2,-2}^{*} \\
& p_{1,-1}^{*} & p_{2,-1}^{*} \\
{[\mathrm{x}]} & p_{1,0}^{*} & p_{2,0}^{*} \\
& p_{1,1}^{*} & p_{2,1}^{*} \\
& p_{1,2}^{*} & p_{2,2}^{*}
\end{array}\right] \text {, }
$$

where the output position again is under the "[x]" coefficient.

The LP system consisting of expressions (13) and (14), with coverage over all $(m, n)$ data points, may be written as

$$
\left[\begin{array}{c}
\vdots \\
\mathbf{a}_{m-1, n}^{T} \\
\mathbf{a}_{m, n}^{T} \\
\vdots \\
\left(\mathbf{b}_{m, n}^{T}\right)^{*} \\
\left(\mathbf{b}_{m+1, n}^{T}\right)^{*} \\
\vdots
\end{array}\right] \mathbf{p}=\left[\begin{array}{c}
\vdots \\
u_{m, n} \\
u_{m+1, n} \\
\vdots \\
u_{m-1, n}^{*} \\
u_{m, n}^{*} \\
\vdots
\end{array}\right]
$$

b)

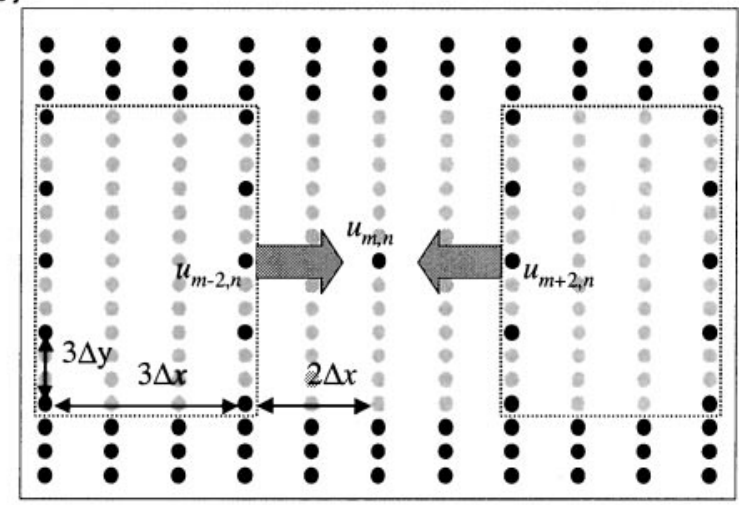

d)

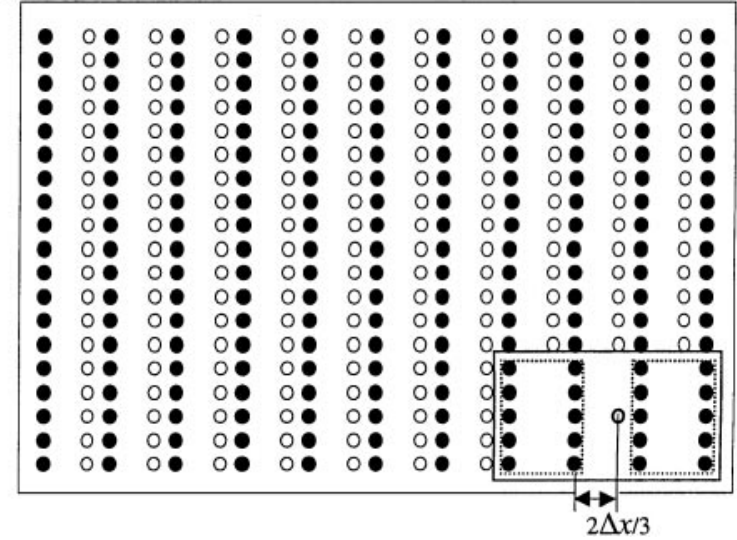

FIG. 5. The fractional step prediction method: (a) the 1/3-step forward and backward predictions; (b) the 2/3-step forward and backward predictions; (c) the interpolated sample $u_{m+1 / 3, n}$ is obtained by the $1 / 3$-step forward and $2 / 3$-step backward predictions; and (d) the interpolated sample $u_{m+2 / 3, n}$ is obtained by the $2 / 3$-step forward and $1 / 3$-step backward predictions. 
The 2/3-step LP operator is then designed using the masked data set shown in Figure 5b. In a similar way, the LP system may be written as

$$
\left[\begin{array}{c}
\vdots \\
\mathbf{a}_{m-1, n}^{T} \\
\mathbf{a}_{m, n}^{T} \\
\vdots \\
\left(\mathbf{b}_{m, n}^{T}\right)^{*} \\
\left(\mathbf{b}_{m+1, n}^{T}\right)^{*} \\
\vdots
\end{array}\right] \mathbf{q}=\left[\begin{array}{c}
\vdots \\
u_{m+1, n} \\
u_{m+2, n} \\
\vdots \\
u_{m-2, n}^{*} \\
u_{m-1, n}^{*} \\
\vdots
\end{array}\right],
$$

where $\mathbf{q}$ is the 2/3-step LP operator. This LP operator $\mathbf{q}$ has the same form as the 1/3-step LP operator $\mathbf{p}$ illustrated in expression (15).

Equations (16) and (17) are solved as one linear system, which may be represented in vector-matrix notation as

$$
\mathbf{D}\left[\begin{array}{ll}
\mathbf{p} & \mathbf{q}
\end{array}\right]=\left[\begin{array}{ll}
\mathbf{d}_{1} & \mathbf{d}_{2}
\end{array}\right]
$$

where $\mathbf{D}$ is the data matrix consisting of $\mathbf{a}_{m, n}$ and $\mathbf{b}_{m, n}$, and $\mathbf{d}_{1}$ and $\mathbf{d}_{2}$ are the desired output vectors. The solution of the LP operators $\mathbf{p}$ and $\mathbf{q}$ are then given by

$$
\left[\begin{array}{ll}
\mathbf{p} & \mathbf{q}
\end{array}\right]=\left(\mathbf{D}^{H} \mathbf{D}+\sigma^{2} \mathbf{I}\right)^{-1} \mathbf{D}^{H}\left[\begin{array}{ll}
\mathbf{d}_{1} & \mathbf{d}_{2}
\end{array}\right]
$$

\section{Trace interpolation}

In the interpolation stage, given $M \times N$ samples input $\left\{u_{m, n}\right\}$, we first interpolate $(M-1) \times N$ samples $\left\{u_{m+1 / 3, n}\right\}$, and then $(M-1) \times N$ samples $\left\{u_{m+2 / 3, n}\right\}$.

The interpolation trace $u_{m+1 / 3, n}$ with trace interval $\Delta x / 3$ to the left line and $2 \Delta x / 3$ to the right line is produced by the $1 / 3$ step forward and the $2 / 3$-step backward predictions, as shown schematically in Figure 5c. The 1/3-step forward prediction may be expressed as

$$
\left[\begin{array}{cc}
u_{m-1, n-2} & u_{m, n-2} \\
u_{m-1, n-1} & u_{m, n-1} \\
u_{m-1, n} & \frac{u_{m, n}}{u_{m-1, n+1}} \\
u_{m-1, n+2} & u_{m, n+2}
\end{array}\right] \rightarrow u_{m+1 / 3, n}
$$

and the 2/3-step backward prediction may be expressed as

$$
u_{m+1 / 3, n} \leftarrow\left[\begin{array}{cc}
u_{m+1, n-2} & u_{m+2, n-2} \\
u_{m+1, n-1} & u_{m+2, n-1} \\
\frac{u_{m+1, n}}{u_{m+1, n+1}} & u_{m+2, n} \\
u_{m+1, n+2} & u_{m+2, n+2}
\end{array}\right],
$$

where the two data matrices can be denoted as vectors $\tilde{\mathbf{a}}_{m, n}$ and $\tilde{\mathbf{b}}_{m+1, n}$. The interpolated trace $u_{m+1 / 3, n}$ is given by the arithmetic average of the forward and backward predictions:

$$
u_{m+1 / 3, n}=\frac{1}{2}\left(\tilde{\mathbf{p}}_{\mathrm{f}}^{T} \tilde{\mathbf{a}}_{m, n}+\tilde{\mathbf{q}}_{\mathrm{b}}^{T} \tilde{\mathbf{b}}_{m+1, n}\right)
$$

where $\tilde{\mathbf{p}}_{\mathrm{f}}$ is the $1 / 3$-step forward LP operator, and $\tilde{\mathbf{q}}_{\mathrm{b}}$ is the 2/3-step backward LP operator. Combining these two operators, the all-azimuth LP operator spanning the $x-y$ space has the form

$$
\frac{1}{2}\left[\begin{array}{ccccc}
\tilde{p}_{2,2} & \tilde{p}_{1,2} & & \tilde{q}_{1,-2}^{*} & \tilde{q}_{2,-2}^{*} \\
\tilde{p}_{2,1} & \tilde{p}_{1,1} & & \tilde{q}_{1,-1}^{*} & \tilde{q}_{2,-1}^{*} \\
\tilde{p}_{2,0} & \tilde{p}_{1,0} & {[\mathrm{x}]} & \tilde{q}_{1,0}^{*} & \tilde{q}_{2,0}^{*} \\
\tilde{p}_{2,-1} & \tilde{p}_{1,-1} & & \tilde{q}_{1,1}^{*} & \tilde{q}_{2,1}^{*} \\
\tilde{p}_{2,-2} & \tilde{p}_{1,-2} & & \tilde{q}_{1,2}^{*} & \tilde{q}_{2,2}^{*}
\end{array}\right] .
$$

The interpolation then is implemented as a spatial convolution.

In a similar way, the interpolation sample $u_{m+2 / 3, n}$ with spatial interval $2 \Delta x / 3$ to the nearest line on the left and $\Delta x / 3$ on the right (as depicted in Figure 5d) is generated by

$$
u_{m+2 / 3, n}=\frac{1}{2}\left(\tilde{\mathbf{q}}_{\mathrm{f}}^{T} \tilde{\mathbf{a}}_{m, n}+\tilde{\mathbf{p}}_{\mathrm{b}}^{T} \tilde{\mathbf{b}}_{m+1, n}\right),
$$

where $\tilde{\mathbf{q}}_{\mathrm{f}}$ and $\tilde{\mathbf{p}}_{\mathrm{b}}$ are the 2/3-step forward and 1/3-step backward LP operators, respectively. The corresponding allazimuth LP operator is expressed explicitly in the following matrix:

$$
\frac{1}{2}\left[\begin{array}{ccccc}
\tilde{q}_{2,2} & \tilde{q}_{1,2} & & \tilde{p}_{1,-2}^{*} & \tilde{p}_{2,-2}^{*} \\
\tilde{q}_{2,1} & \tilde{q}_{1,1} & & \tilde{p}_{1,-1}^{*} & \tilde{p}_{2,-1}^{*} \\
\tilde{q}_{2,0} & \tilde{q}_{1,0} & {[\mathrm{x}]} & \tilde{p}_{1,0}^{*} & \tilde{p}_{2,0}^{*} \\
\tilde{q}_{2,-1} & \tilde{q}_{1,-1} & & \tilde{p}_{1,1}^{*} & \tilde{p}_{2,1}^{*} \\
\tilde{q}_{2,-2} & \tilde{q}_{1,-2} & & \tilde{p}_{1,2}^{*} & \tilde{p}_{2,2}^{*}
\end{array}\right] .
$$

The description above applies to interpolation with fractional increments such as $1 / 3$ and $2 / 3$. For the case with interpolation factor $\lambda=2$, only one LP operator (referred to as the half-step LP operator) is needed. In that case, equation (18) may be used in the estimation stage, but the operators $\mathbf{p}$ or $\mathbf{q}$ are then identical in the resulting linear system. An all-azimuth LP operator similar to expression (23) [or expression (25)], in which $\tilde{p}_{i, j}$ is equivalent to $\tilde{q}_{i, j}$, is then used to interpolate the $(M-1) \times N$ new traces. In the case $\lambda=4$, instead of $1 / 4-, 1 / 2-$, and 3/4-step LP interpolations, it is simplest and preferable to perform the half-step LP interpolation twice.

\section{DISCUSSION AND EXAMPLES}

The full-step and the fractional-step linear prediction methods both are based on the LP theory but differ in the implementation. The full-step prediction method requires solution of two linear systems: one in the estimation stage [equation (8)] and one in the interpolation stage [equation (12)]. The second of these linear systems takes intensive computational time to resolve. The fractional-step prediction method, however, requires solution of only one linear system [equations (18) in the estimation stage], whereas the interpolation is accomplished by the simple convolution [equations (22) and (24)]. Thus, the fractional-step prediction method is much faster than the fullstep method.

For design of an $L \times L$ operator with $L=5$ and $\lambda=3$, for example, one needs at least $5 \times 13$ traces for the full-step prediction (Figure 3), but $9 \times 13$ traces for the $1 / 3$-step prediction 
and $11 \times 13$ traces for the $2 / 3$-step prediction (Figure 5). If we assume that $\Delta x=3 h$ and $\Delta y=h$, the supporting area would be a $12 h \times 12 h$ square for the full-step prediction filter and a $30 h \times 12 h$ rectangle for the fractional step prediction filter. Based on several experiments that I have conducted, however, the difference between the interpolation results of these two methods is tolerable. Therefore, the fractional-step method is used in the following examples.

To demonstrate the method, 3-D synthetic traces with a diffraction hyperbola are generated. The data cube consists of 301 lines (numbered from 1 to 301), and each line has 301 CDPs (numbered from 850 to 1150). Each seismic trace is 4-s long with a sample interval of $4 \mathrm{~ms}$. The diffraction hyperbola is generated from a spike at time $1 \mathrm{~s}$ and at line 151 and CDP 1000. The interpolation program doesn't require the specification of spatial intervals, as long as they are regular in each direction. To demonstrate the trace interpolation, every third line from the synthetic is selected to form an input cube.

The interpolation program is then used to generate two new lines between each pair of input lines (i.e., $\lambda=3$ ). Figure $6 \mathrm{a}$ displays an interpolated line (101), for comparison with the original synthetic traces shown in Figure 6b. The difference between the interpolated and decimated traces is highlighted in Figure $6 \mathrm{c}$. Since it is a linear prediction method, Figure $6 \mathrm{c}$ shows the greatest amplitude difference where the event curvature is greatest. Interpolation with $\lambda=2$ has much less amplitude residual than the case shown here with $\lambda=3$.

A real data example of trace interpolation is shown in Figure 7, where every second gather is the interpolated shot gather interleaved with the original shot gathers (i.e., $\lambda=2$ ). The two spatial axes in this case refer to the shot coordinate and the source-receiver offset. If a $f-x$ rather than the $f-x$ $y$ interpolator was applied within a shot gather, representation of curved events would have difficult. The $f-x-y$ domain prediction, however, can relax the requirement that events be linear (Chase, 1992; Abma and Claerbout, 1995; Gülünay, 2000). Events that are nonlinear in one direction but linear in another may be predicted exactly with the $f-x-y$ prediction filter.

\section{CONCLUSION}

The so-called $f-x-y$ domain trace interpolation is implemented as the 2-D spatial prediction on frequency slices. The methodology exploits the relationship that the prediction operator estimated at a given frequency may be used to predict data at a higher frequency but a smaller trace spacing. This relationship given originally for the $f-x$ domain trace interpolation is extended successfully to the $f-x-y$ domain. The extension is achieved by masking the original data samples selectively when designing the linear prediction operator on a frequency slice. In both, the two interpolation algorithms, using either the full-step or the fractional-step prediction theorems, the LP operator is designed as a combination of forward and backward predictions. This strategy is attractive in computation because of the conjugate symmetry in the resultant all-azimuth operators. Although both algorithms are an all-azimuth linear prediction in the $x$ - $y$ space, the fractional-step prediction method is more efficient computationally than the full-step method, despite that the efficiency of the latter method has been improved by constructing the interpolation within divided subblocks.

\section{ACKNOWLEDGMENTS}

Thanks to S. Merrylees for his support to this project; to R. Abma, S. Ronen, and A. Strudley for reviewing the manuscript; and to Professor G. Houseman of Leeds University for a finalized review. The paper is published with permission of Robertson Research International Ltd.

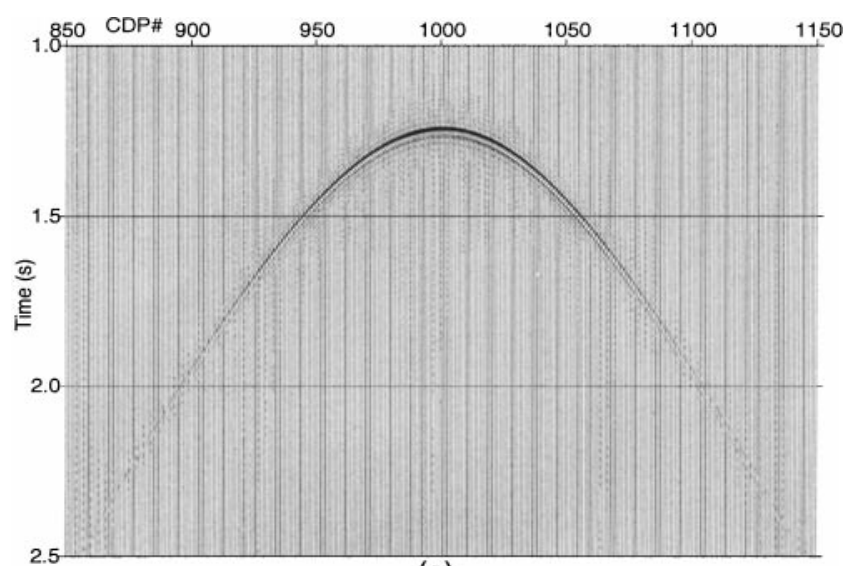

(a)

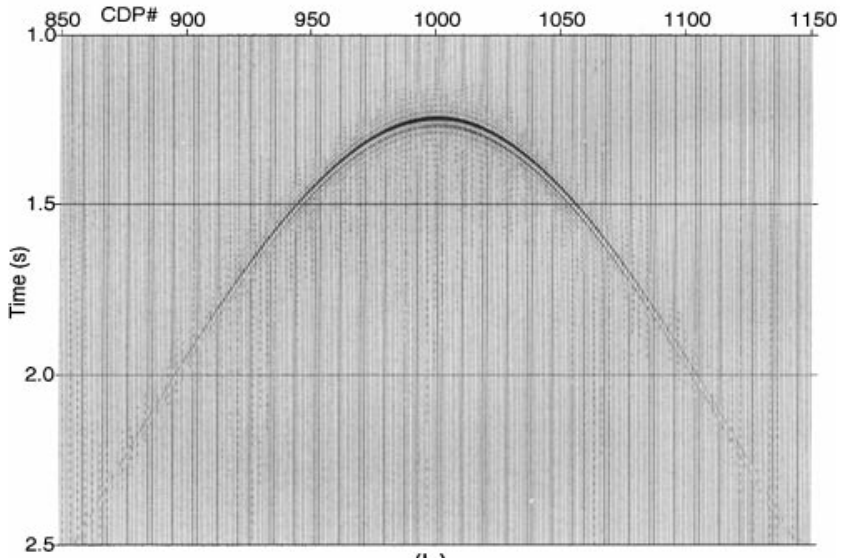

(b)

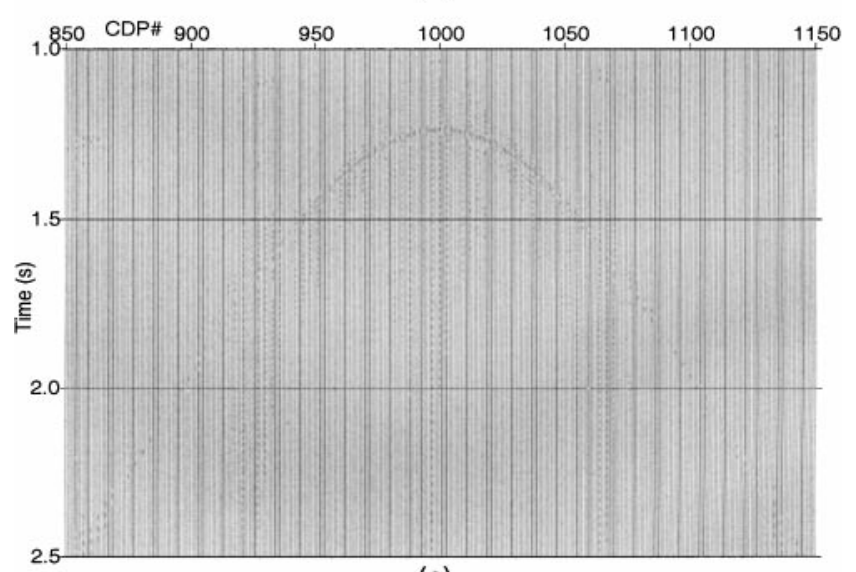

(c)

FIG. 6. Trace interpolation example: (a) an in-line section obtained by trace interpolation with $\lambda=3$; (b) the corresponding in-line section from the original synthetic data set; (c) the difference between the interpolation traces and the synthetics, shown with the same amplitude scale. 


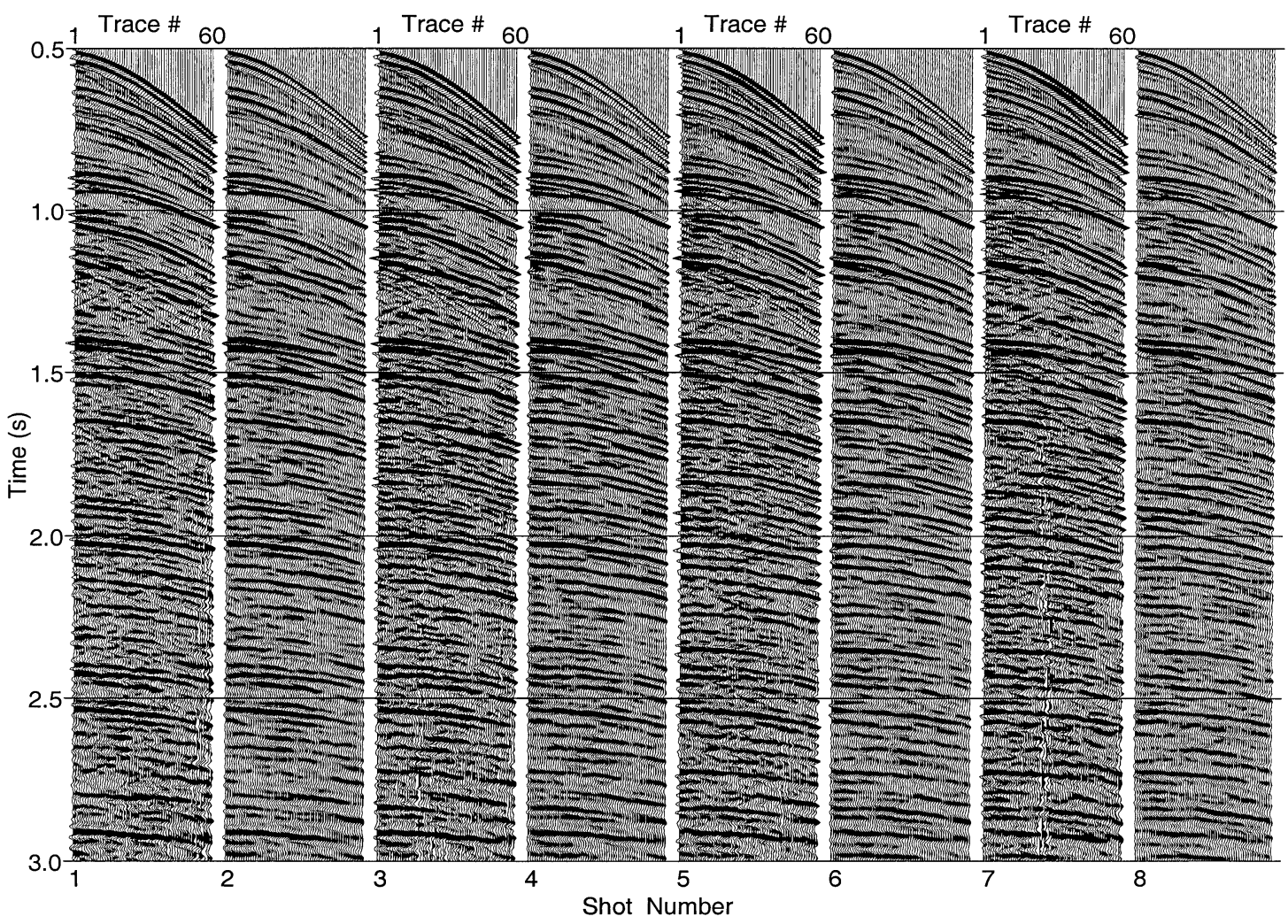

FIG. 7. Trace interpolation example. Newly interpolated shot gathers are interleaved between the raw shot gathers. The interpolation factor used in this example is $\lambda=2$. Odd-numbered shot gathers are original data, and even-numbered gathers are interpolated.

\section{REFERENCES}

Abma, R., and Claerbout, J., 1995, Lateral prediction for noise attenuation by $t-x$ and $f-x$ techniques: Geophysics, $\mathbf{6 0}, 1887-1896$.

Bardan, V., 1987, Trace interpolation in seismic data processing: Geophys. Prosp., 35, 343-358.

Canales, L. L., and Lu, L., 1993, 3-D trace interpolation in the $f-x-p$ domain: 63rd Ann. Internat. Mtg., Soc. Expl. Geophys., Expanded Abstracts, 1174-1176.

Chase, M. K., 1992, Random noise reduction by $F X Y$ prediction filtering: Expl. Geophys., 23, 51-56.

Claerbout, J. F., 1992, Earth soundings analysis: Processing versus inversion: Blackwell Scientific Publications.
Gülünay, N., 2000, Noncausal spatial prediction filtering for random noise reduction on 3-D poststack data: Geophysics, 65, 16411653.

Larner, K., Gibson, B., and Rothman D., 1981, Trace interpolation and the design of seismic survey: Geophysics, 46, 407.

Porsani, M. J., 1999, Seismic trace interpolation using half-step prediction filters: Geophysics, 64, 1461-1467.

Spitz, S., 1991, Seismic trace interpolation in the $F-X$ domain: Geophysics, 56, 785-794.

Van Der Vorst, H. A., and Dekker, K., 1988, Conjugate gradient type methods and preconditioning: J. Comp. Appl. Math., 24, 73-87.

Wang, Y., 1999, Random noise attenuation using forward-backward linear prediction: J. Seismic Expl., 8, 133-142. 